\title{
VOLUME OF FLUID SIMULATION OF BOILING TWO-PHASE FLOW IN A VAPOR-VENTING MICROCHANNEL
}

\author{
Chen Fang", Milnes David, Anita Rogacs, Kenneth Goodson \\ Mechanical Engineering Department, Stanford University, Stanford, CA, 94305
}

\begin{abstract}
Vapor-venting microchannel heat exchangers are promising because they address the problems of high pressure drop, flow instability, and local dryout that are common in conventional two-phase microchannel heat sinks. We present a 3D numerical simulation of the vapor-venting process in a rectangular microchannel bounded on one side by a hydrophobic porous membrane for phase-separation. The simulation is based on the volume of fluid (VOF) method together with models for interphase mass transfer and capillary force. Simulation shows the vapor-venting mechanism can effectively mitigate the vapor accumulation issue, reduce the pressure drop, and suppress the local dry-out in the microchannel. Pressure surge is observed in the vapor-venting channel. The simulation provides some insight into the design and optimization of vapor-venting heat exchangers.

Keywords: Volume of Fluid, Porous Membrane, Capillary-induced Phase Separation
\end{abstract}

\section{INTRODUCTION}

Continuing increases in component density and reductions in VLSI chip dimensions demand the development of high-performance and low-profile thermal packaging solutions. According to the International Technology Roadmap for Semiconductors high performance microprocessors require heat removal rates exceeding $200 \mathrm{~W}$ with a thermal resistance of up to $0.1 \mathrm{~K} / \mathrm{W}$ (David et al., 2007). Conventional cooling techniques based on fans and heat pipes do not scale well with diminished device dimensions. Several studies (Tuckerman et al.; Qu et al., 2002; Kandlikar et al., 2004; Garimella et al., 2004) showed that single-phase liquid convection in microchannels can dissipate large heat fluxes only at the expense of high pumping power. Two-phase boiling flow in microchannels promises low thermal resistances with low pumping power requirements. However several drawbacks make two-phase heat sinks less attractive at present. These include flow instabilities (Kennedy et al., 2000), high peak pressure drops (Zhang et al., 2005; Garimella et al., 2002), and dry-out, which causes hotspots. These issues are at least partially attributed to the excessive vapor phase accumulation in the microchannel during the boiling process.

One proposed solution to the vapor phase accumulation, put forth by Zhou et al. (2006), is to locally vent the vapor phase generated in the system into a separate channel, leaving the liquid channel primarily occupied by the liquid phase. The vapor-escape mechanism reduces the two-phase pressure drop to pressures seen in systems of single-phase microchannels while maintaining the high heat fluxes associated with the phase change process. The removal of the vapor phase may also minimize the vapor-expansion-induced flow instability and the dry-out caused by the local liquid film rupture.

The core technological challenge of the vapor-venting heat exchanger lies in achieving phase separation that is reliable and leakage free for the liquid phase. The first experimental realiziation of this approach was provide by David et al. (2007). The design, as illustrated in Fig.1, incorporates a pair of parallel liquid and vapor silicon microchannels sandwiching a porous, hydrophobic membrane inbetween. A similar approach was applied to microdirect methanol fuel cells for the reaction byproduct removal (Meng et al., 2004; Meng et al., 2007). An adiabatic test using an air-water mixture showed an airmass venting efficiency exceeding $95 \%$ and a significant pressure drop compared to the non-venting model. However, the opaque vaporventing membrane and a silicon substrate made the flow pattern visualization in the microchannel unavailable. A solution to that issue is using a quartz substrate and/or a semi-transparent membrane. As an alternative to the silicon-based vapor venting heat exchanger, David et al. (2008) developed vapor-venting copper heat exchangers with integrated hydrophobic PTFE membranes. However, no overall reduction in thermal resistance or pressure-drop was observed in the open venting device. The analysis attributed this to the water saturation of the carbon-fiber membrane that drastically reduced the venting area. Similar to silicon-based devices, the copper heat exchanger lacked the optical access, making it hard to study the vapor-venting mechanism. More recently, visualization of the gas-venting process was achieved by David et al. (2009) and Alexander et al. (2008) using a porous hydrophobic Teflon membrane and microfabricated hydrophobic trenches for phase separation, respectively. Both studies were based on an adiabatic system involving the air-water mixture and a single air injection site emulating the boiling nucleation. Although those studies yielded valuable information about the flow pattern and vapor-venting effectiveness, the coupling between heat transfer and flow were not considered.

Transient 3D numerical simulations based on the Volume Of Fluid (VOF) method provide an alternative way to explore the bubble dynamics and venting mechanism, which is essential for both understanding the underlying physics of the vapor-venting process and device optimization purposes. Unlike many other numerical methods treating liquid and vapor phase separately, the VOF method dynamically tracks the interface and requires no knowledge of the interface location (e.g., Fang et al., 2008). Yang et al. (2008) performed VOF simulations of boiling flow of R141B in a horizontal

*Corresponding author, Email: chenfang@stanford.edu 
coiled tube. The calculations treated the phase change as a local temperature dictated process based on a quasi thermo-equilibrium assumption, which yielded a flow pattern in good agreement with the experimental observation. Zhang et al. (2001) relaxed the thermoequilibrium assumption and treated the condensation as a process dictated by the heat transfer rate across the interface. However, the model requested an artificial source term to be added to the enthalpy equation to keep the liquid-vapor interface temperature at the saturation point. The VOF method was also used to model other types of phase change process, such as boiling (Welch et al., 2000), solidification (Takata et al., 1999), and laser-induced vaporization (Ganesh et al., 1997). The phase change process in the porous structure poses a new challenge to the modeling due to the interaction between porous media and fluid. Most previous studies utilized either the pore network model (Figus et al., 1999) or the finite element model which treats the liquid and vapor zone separately while coupling two phases by imposing a pressure jump on the interface (Kaya and Goldak, 2006; Ren et al., 2007; Figus et al., 1999). Alternatively, VOF method dramatically facilitates the interface tracking. However, the capillary force associated with the multiphase flow in porous media needs to be properly modeled within the VOF framework.

We use the VOF method to study 3D boiling flow in a rectangular microchannel with a hydrophobic vapor-venting membrane on one boundary. The phase separation is modeled using a capillary force model. The simulation illustrates the impact of applied heat flux, membrane hydrophobicity, and channel geometry on the flow pattern, pressure drop, and temperature profile in the vapor-venting microchannel. A comparison between the conventional channel and the vapor-venting channel clearly shows the pros and cons of the new design. The simulation result provides a tool for designing and optimizing the next generation vapor-venting micro heat exchanger.

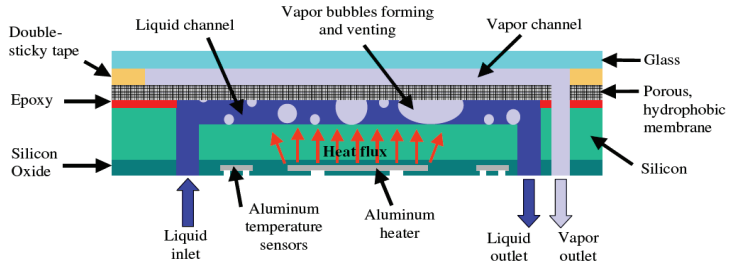

(a) Schematic of a vapor-venting microchannel

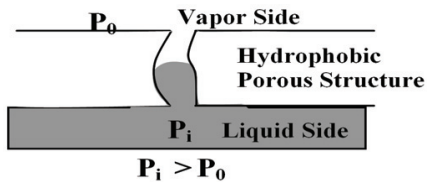

(b) Liquid containment enabled by a hydrophobic membrane

Fig. 1 Schematic of the vapor-venting microchannel design and the liquid-vapor transport through a hydrophobic porous membrane

\section{PHYSICAL MODEL}

\subsection{Governing Equations}

The VOF method uses one set of governing equations to model both the liquid and vapor region, which substantially facilitates the interface tracking and interfacial force treatment. The flow is modeled with the Navier-Stokes equation:

$\rho\left(\frac{\partial u}{\partial t}+(u \cdot \nabla) u\right)=-\nabla p+\nabla \cdot(\mu S)+f$

where $\rho, \mu$ and $f$ are the density, viscosity, and body force term, respectively. The rate of strain tensor $S$ can be expressed as:
$S_{i j}=\frac{1}{2}\left(\frac{\partial u_{j}}{\partial x_{i}}+\frac{\partial u_{i}}{\partial x_{j}}\right)$

The velocity field $u$ is subject to the incompressibility constraint

$\nabla \cdot u=0$

VOF method defines the interface separating immiscible liquids of different density and viscosity based on a phase indicator - the volume fraction function $\alpha$. To allow for mass conservation of each phase, an additional equation is introduced:

$$
\frac{\partial \alpha}{\partial t}+u \cdot \nabla \alpha=\operatorname{Src}
$$

where the volumetric source term Src dictates the phase change rate. We use (4) to represent and track the interface which is transported by the velocity field $u$. The energy equation is written as:

$\partial(\rho E) / \partial t+\nabla \cdot[u(\rho E+p)]=\nabla \cdot(K \nabla T)+Q$

where $Q$ represents the phase change induced energy transfer, which is computed from the evaporation mass rate $\operatorname{Src}$ and the latent heat $h$ :

$Q=-h S r c$

The density, viscosity, thermal conductivity, and energy at the interface are calculated using the volume fraction $\alpha$ :

$$
\begin{aligned}
& \rho=\alpha \rho_{l}+(1-\alpha) \rho_{v} \\
& \mu=\alpha \mu_{l}+(1-\alpha) \mu_{v} \\
& K=\alpha K_{l}+(1-\alpha) K_{v} \\
& E=\frac{\left(\alpha \rho_{l} C_{v, l}+(1-\alpha) \rho_{v} C_{v, v}\right)\left(T-T_{s a t}\right)}{\rho}
\end{aligned}
$$

The current VOF model employs the Marker and Cell (MAC) finite difference method based on rectangular grids. The volume fraction $\mathrm{C}$ and the pressure $p$ are defined at the center of each cell, while the horizontal and vertical velocity components are defined at the midpoints of the vertical and horizontal sides, respectively. At each time step the volume fraction $\alpha$, which indicates the two-phase interface position, is first updated followed by the evaluation of the surface tension force. Finally, the velocity and pressure fields are solved using Eq.(1).

The interface location is updated using the Young's piecewise linear interface construction (PLIC) scheme (Youngs, 1982), in which the normal vector $n$ of the interface is first related to the gradient of volume fraction $C$ by $n=\nabla C /|\nabla C|$. Then, a flat plane having a normal vector $\mathrm{n}$ is used to approximate the real liquid-gas interface within each cell. With the knowledge of the interface position change, the volume of fluid transferring into the neighboring cells is evaluated and the volume fraction $\alpha$ updated. Due to the small Reynolds number which is typical for microscale flows, a laminar flow model is used. The equations are solved by an algebraic multigrid (AMG) solver (Issa et al., 1991), and the pressure-implicit with splitting of operators (PISO) scheme for pressure-velocity coupling (Brackbill et al., 1992).

\subsection{Surface Tension in Channel Region}

Interfacial surface tension arises as the result of intermolecular attractive forces. The Young-Laplace equation expresses the surface tension in terms of the surface curvature $k$, surface tension coefficient $\sigma$, and the surface normal $n$ :

$f=\sigma k n$

In our simulation, surface tension is modeled using the Continuous Surface Force (CSF) scheme (Brackbill et al., 1992). The surface tension is modeled by a source term $f$ in the momentum equation:

$f=\sigma k \frac{2 \rho}{\rho_{1}+\rho_{2}} \nabla \alpha$

where $\rho$ is given by (7). The density $\rho$ in Eq.(12) inhibits the numerical smearing of the interface and is elaborated by Brackbill et 
al.(1992). In the present study, Maragoni effects are not considered, therefore $\sigma$ is treated as constant. The curvature $k$ is defined in terms of the divergence of the unit normal $\hat{n}$ :

$k=-(\nabla \cdot \hat{n})=-\left[\nabla \bullet\left(\frac{1}{|\nabla \alpha|} \nabla \alpha\right)\right]$

where

$\hat{n}=\frac{n}{|n|}$

Substituting (13) into (12) yields:

$$
f=2 \sigma \rho \frac{\nabla \alpha}{\rho_{l}+\rho_{g}} \frac{1}{|\nabla \alpha|}\left[\left(\frac{\nabla \alpha}{|\nabla \alpha|} \bullet \nabla\right)|\nabla \alpha|-\left(\nabla^{2} \alpha\right)\right]
$$

In applying formulation (15), a Marker and Cell (MAC) like discretization scheme is used. A unit vector $n$ is calculated from discretizing the volume fraction $\alpha$ of the immediate 26 neighboring cells, based on the relation $n=\nabla \alpha$. Initially, a cell corner value of the normal vector is computed. Then required cell-centered values are obtained by averaging the corner values. Also, $\nabla^{2} \alpha$ is calculated by discretizing the normal vector values obtained at the eight cell corners.

\subsection{Flow in Porous Membrane}

Unlike in the channel region, flow field in the porous region is described by the superficial velocity $u_{s}$ defined based on the volumetric flow rate instead of the real physical velocity. The incompressibility constraint yields:

$\nabla \cdot u_{s}=0$

For a low flow velocity, we have a laminar flow with a negligible inertial force. The momentum equation reads:

$-\nabla P=\frac{\mu}{K} u_{s}-f_{\text {capillary }}$

which assumes homogeneous, isotropic porous media giving a constant permeability $K . f_{\text {capillary }}$ represents the capillary force acting on the liquid-vapor interface. Unlike the surface tension force on the liquidvapor interface in the channel region, the capillary force in the porous media represents the interaction between the porous structure and a set of small meniscuses on the order of the pore size, and is therefore independent of the macroscopic curvature of the interface. By replacing the $\sigma k$ term in (12) with the capillary pressure jump $P_{\text {capillary }}$ across the interface, we have:

$f_{\text {capillary }}=P_{\text {capillary }} \frac{2 \rho}{\rho_{1}+\rho_{2}} \nabla \alpha$

The capillary pressure $P_{\text {capillary }}$ depends on the pore size and the wettability of the porous structure. A simplified model treating the porous structure as a bundle of capillary tubes gives the equation:

$P_{\text {capillary }}=\frac{4 \sigma_{f} \cos \theta}{D}$

where $D$ is the average pore size and $\sigma_{f}$ is the surface energy. The equilibrium contact angle $\theta$ is a measure of the porous media wettability. A hydrophobic porous membrane with large $\theta$ leads to a capillary force pointing toward the liquid phase, preventing the liquid leakage through the porous media. In this study, the venting membrane is treated as a homogeneous porous media, therefore $P_{\text {capillary }}$ is independent of the orientation and the location of the meniscus.

The energy equation in the porous region takes the form:

$\frac{\partial\left[\varphi \rho_{f} E_{f}+(1-\varphi) \rho_{s} E_{s}\right]}{\partial t}+\nabla \cdot\left[u_{s}\left(\rho_{f} E_{f}+P\right)\right]=\nabla \cdot\left(k_{e f f} \nabla T\right)+Q$
Equation (20) assumes an isotropic and homogeneous effective thermal conductivity $k_{\text {eff }}$, which can be obtained based on the membrane porosity $\varphi$ :

$k_{\text {eff }}=\varphi k_{f}+(1-\varphi) k_{s}$

In Eqs. (20) and (21), the fluid properties $\rho_{f}, k_{f}$, and energy $E_{f}$ are determined by Eqs. (7), (9), (10), respectively. The energy source term $Q$ is calculated by Eq. (6).

\subsection{Phase Change Model}

Yang et al. (2008) used a mass transfer model proposed by Lee (1980) to simulate the two-phase boiling flow in a coiled tube. By assuming that phase change occurs at a quasi thermo-equilibrium state at a constant pressure and that the phase change rate $S$ is mainly dictated by the local temperature $T_{l}$, the saturation temperature $T_{\text {sat }}$, and the volumetric fraction of the liquid/vapor phase $\alpha_{l}$ and $\alpha_{v}$, we have:

$\operatorname{Src}=r_{l} \alpha_{l} \rho_{l}\left(T_{l}-T_{\text {sat }}\right) / T_{\text {sat }} \quad T_{l} \geq T_{\text {sat }}$ Src $=r_{v} \alpha_{v} \rho_{v}\left(T_{l}-T_{\text {sat }}\right) / T_{\text {sat }} T_{l}<T_{\text {sat }}$

Yang et al. (2008) recommends setting the empirical coefficient $r_{l}$ and $r_{v}$ to be $100 s^{-1}$ in order to numerically maintain the interface temperature at $T_{\text {sat }}$, and the choice was justified by a good agreement between the model prediction and the experiment. Too large $r_{l}$ and $r_{v}$ induces a numerical oscillation, whereas excessively small values cause the interfacial temperature to substantially deviate from $T_{\text {sat }}$. In the present study, the source term Src in Eq. (4) is evaluated using Eq. (22), where $\alpha_{l}$ and $\alpha_{v}$ can be obtained within the framework of VOF model. An alternative method of regulating the interface temperature based on artificial parameters was discussed by Zhang et al. (2001).

\subsection{Geometric Configuration and Boundary Conditions}

In the present study, a straight channel with a rectangular cross-section is considered. A vapor-venting porous membrane covers the top of the channel. A uniform heat flux is applied to the bottom and two sidewalls. All other walls are set to be adiabatic. A velocity and a pressure boundary condition are assigned to the channel inlet and outlet, respectively. Pure liquid water is pumped into the channel at a temperature around $3 \mathrm{~K}$ below the saturation point, creating a subcooling boiling environment. The membrane-channel interface has a no-slip boundary condition and an effective contact angle, while the other side of the membrane has a pressure boundary condition. The geometry of the simulation domain is shown in Fig. 2 and the parameters are listed in Table 1.

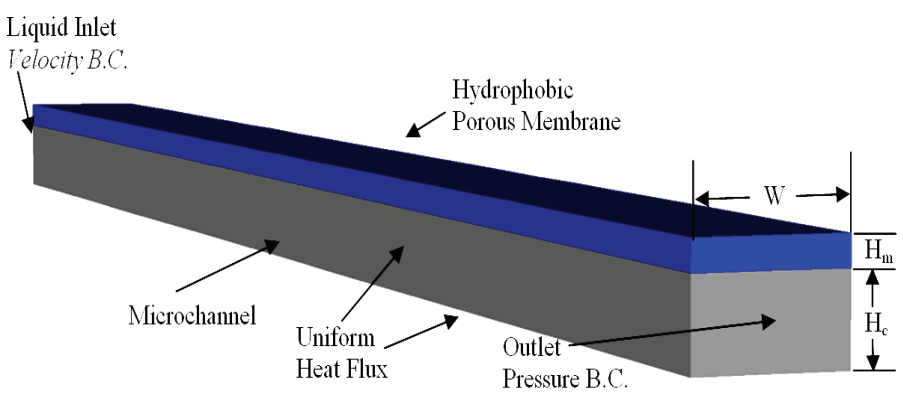

Fig. 2 Computational domain and boundary conditions

Models discussed in Section 2.1-2.4 are applied to the channel and membrane region to account for a variety of physical processes. The phase change model and surface tension model are assigned to the channel domain, while the capillary force model is employed in the 
membrane region to simulate the capillary-induced phase separation. The VOF method has been employed in commercial package FLUENT to simulate multiphase flow with a free surface and is used in the present study. However, the modeling of surface tension acting on the liquid-vapor interface in the microchannel (Section 2.2), the capillary force model (Section 2.3), and the phase change model (Section 2.4) are implemented using a customized user defined code, which is hooked up with the main VOF code to create a closed form model.

In the present study, a 3D structured orthogonal grid with about 191,400 computation nodes was employed. The grid independency was validated by performing different numerical simulations for one particular combination of heat flux, inlet velocity, and outlet pressure with varying number of nodes. This original grid was refined twice by increasing the number of cells by $20 \%$ and $40 \%$ in every direction leading to a $95 \%$ and $200 \%$ finer grid, respectively. Simulation results showed that the difference between them is minor. Hence, the original computational grid with 191,400 was adopted for simulation cases. The mesh statistics are listed in Table 1.

Table 1 Input parameters for the present vapor-venting modeling

\begin{tabular}{|c|c|}
\hline Parameter & Value \\
\hline Channel Length $(\mathrm{mm})$ & 5 \\
\hline Channel Width $(\mu m)$ & 300 \\
\hline Channel Height $H_{c}(\mu m)$ & 150 \\
\hline Membrane Thickness $H_{m}(\mu m)$ & 50 \\
\hline Membrane Permeability-Liquid $\left(m^{2}\right)$ & $4 e-22$ \\
\hline Membrane Permeability-Vapor $\left(m^{2}\right)$ & $8.8 \mathrm{e}-15$ \\
\hline Membrane Contact Angle (deg) & 140 \\
\hline Membrane Pore Size $(\mu m)$ & 0.24 \\
\hline Capillary Pressure $(\mathrm{Pa})^{*}$ & $8.9 \mathrm{e} 5$ \\
\hline Membrane Porosity & 0.6 \\
\hline Surface Heat Flux $\left(\mathrm{Wm}^{-2}\right)^{* *}$ & Varying \\
\hline Liquid Inlet Velocity $(m / s)$ & 0.27 \\
\hline Outlet Gauge Pressure $(\mathrm{Pa})$ & Varying \\
\hline Membrane Outer Surface Gauge Pressure $(\mathrm{Pa})$ & 0 \\
\hline Mesh Size-Channel Space (L x W x H) & $330 \times 20 \times 25$ \\
\hline Mesh Size-Membrane Space (L x W x H) & $330 \times 20 \times 4$ \\
\hline Inlet Liquid Temperature $(\mathrm{K})$ & 370 \\
\hline
\end{tabular}

\section{VALIDATION OF CAPILLARY FORCE MODEL}

The phase change and the surface tension models used in the present study have been validated in previous literatures (Brackbill et al., 1992; Yang et al., 2008). The newly proposed capillary force model in Eq. (18) is validated with a study of the capillary inhibition process into a straight porous cylinder. The water inhibition into the initially dry porous media is driven by the assigned capillary pressure $P_{\text {capillary }}$ of

$700 \mathrm{~Pa}$. Figure 3(a) illustrates a snapshot of the pressure profile in the porous cylinder at time $3.5 \mathrm{e} 4 \mathrm{~s}$. The modeling prediction of capillaryinduced pressure jump across the air-liquid interface agrees well with the specified $P_{\text {capilary }}$. For comparison, a simple 1-D analytical equation can be used to describe the capillary transport in the domain:

$\rho \varepsilon h \frac{d^{2} h}{d t^{2}}+\rho \varepsilon\left(\frac{d h}{d t}\right)^{2}+\frac{\mu}{K} h \frac{d h}{d t}+\rho g \varepsilon h-P_{c a p}=0$ where $\mathrm{h}$ is the liquid column height, $\rho, \mu, K, \varepsilon$ are the liquid density, dynamic viscosity, permeability, and porosity, respectively. Equation (23) can be readily solved using the 4th order Runge-Kutta method and the solution is compared with the current VOF model prediction, as shown in Fig.3(b). A perfect match for liquid rise velocity is obtained for the whole imbibition process, indicating that the current model can correctly capture the dynamics of capillary transport in porous media

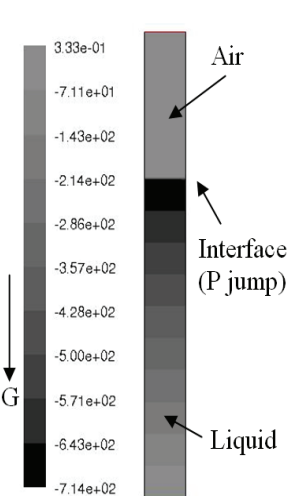

(a) Pressure profile at $\mathrm{t}=3.5 \mathrm{e} 4 \mathrm{~s}$

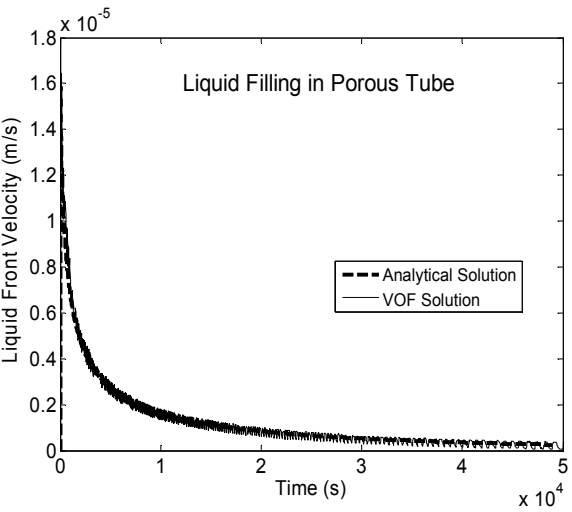

(b) Liquid rise velocity
Fig. 3 Calculated pressure profile and liquid rise velocity for the simulation of water rise in a vertical porous tube

\section{RESULTS AND DISCUSSION}

\subsection{Flow Pattern Overview}

The vapor venting mechanism dramatically changes the mixture quality distribution along the channel, which in turn exerts a significant impact on the flow pattern. Figure 4 shows the comparison of flow patterns between a vapor-venting channel and a conventional channel for heat fluxes ranging from $10 \mathrm{~W} / \mathrm{cm}^{2}$ to $23 \mathrm{~W} / \mathrm{cm}^{2}$, in which the left and right column correspond to the vapor-venting and conventional channel, respectively. To make the two channels comparable, identical initial condition and boundary condition are assigned to both channels except that the vapor-venting channel has its top solid wall replaced by a hydrophobic porous membrane.

For a low heat flux of $10 \mathrm{~W} / \mathrm{cm}^{2}$, as shown in Fig. 4(a), the upstream of both channels is dominated by a bubbly flow. Simulation shows that the bubble size increases in the streamwise direction with the liquid phase temperature. For a vapor bubble attached to a superheated surface, the evaporation occurs near the bubble base (Fig.5 region A) which supplies the vapor to the bubble, while the bubble top interface contacting the subcooled liquid (Fig.5 region B) is dominated by the condensation consuming the vapor phase. The bubble size at equilibrium is dictated by the balance between boiling and condensation, and increases with the bulk liquid temperature. A relevant modeling work for this process was done by Li et al. (2002). The occurrence of vapor venting requires that the bubble grows to a certain size and touch the channel top wall. Hence, the small equilibrium bubble size in the upstream suppresses the vapor-venting, making upstream flow patterns in both channels similar. Small bubbles reaching the departure size may detach from their nucleation sites and be convected to the downstream. Those convected bubbles, nevertheless, tend to move towards the center of the channel due to the Fahraeus-Lindquist effect (Lee et al. 2004), and therefore never touch the membrane surface for venting. In the downstream region, however, a large equilibrium size tends to induce the coalescence of neighboring bubbles, resulting in a vapor venting process (Fig.4(a1) 4(a4)). In contrast, over-sized bubbles in the ordinary channel coalesce and form elongated slugs (Fig.4(a6) 4(a8)), which significantly reduces the cross-sectional area available for the liquid flow. 


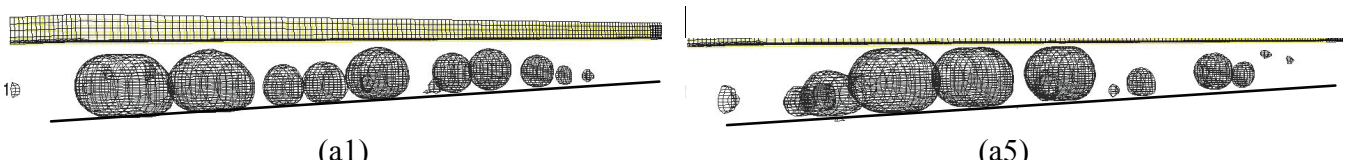

(a1)

$\mathrm{t}=2.3532 \mathrm{e}-2$

(a5)

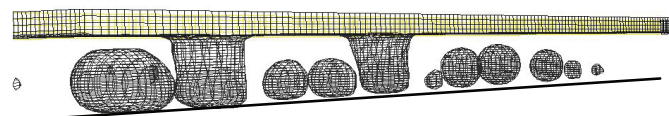

(a2)

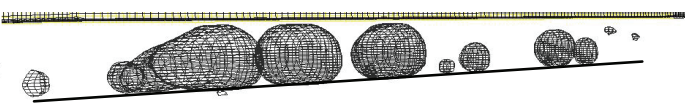

(a6)

$\mathrm{t}=2.3582 \mathrm{e}-2$

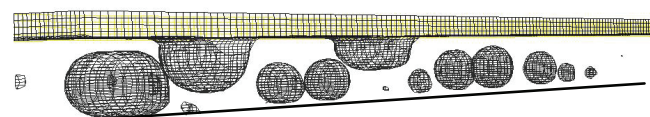

(a3)

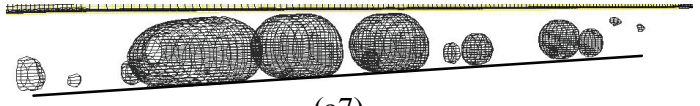

(a7)

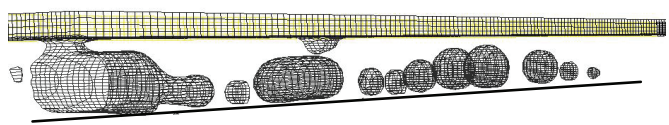

(a4)

$\mathrm{t}=2.3957 \mathrm{e}-2$

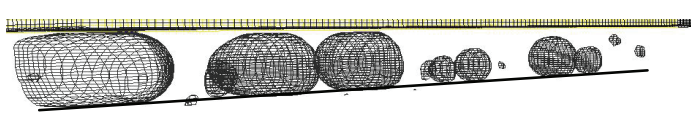

(a8)

(a) Case 1: $10 \mathrm{~W} / \mathrm{cm}^{\wedge} 2$

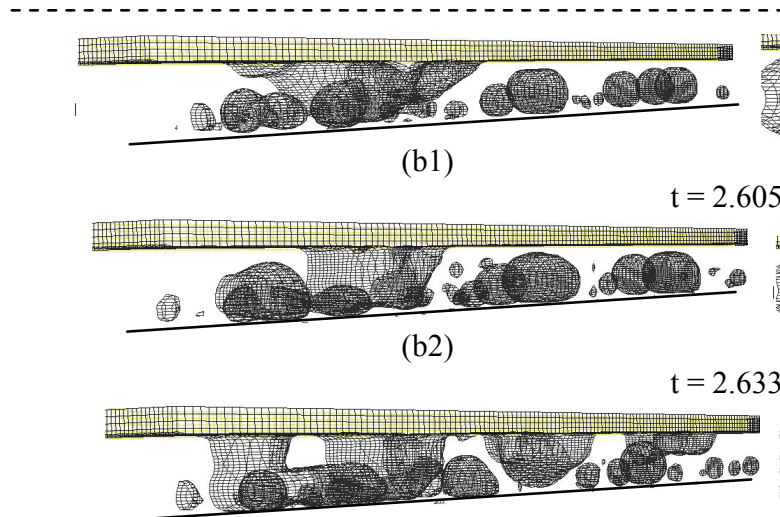

(b3)

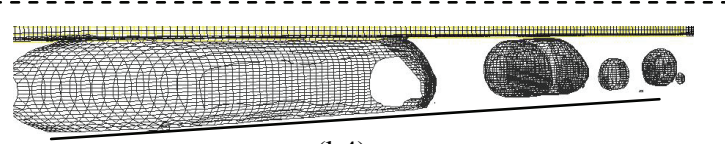

(b4)

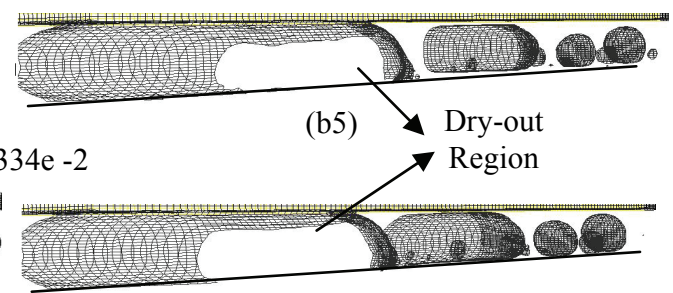

(b6)

(b) Case 2: $18 \mathrm{~W} / \mathrm{cm}^{\wedge} 2$

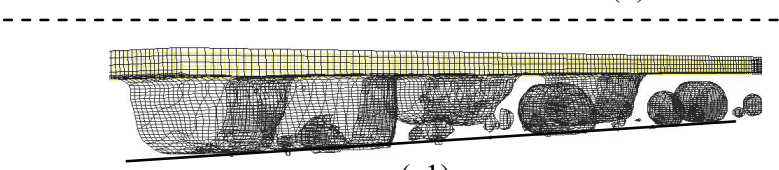

(c1)

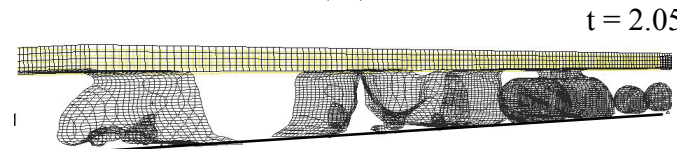

(c2)

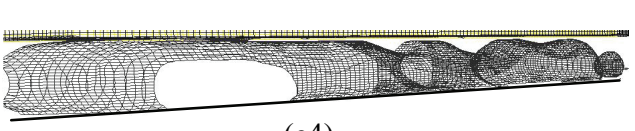

(c4)

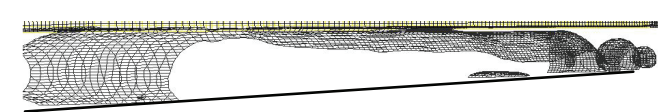

(c5)

$\mathrm{t}=2.1332 \mathrm{e}-2$

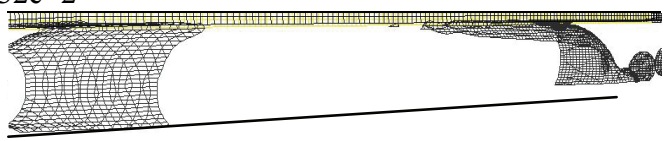

(c6)

$\mathrm{t}=2.2932 \mathrm{e}-2$

(c)Case 3: $23 \mathrm{~W} / \mathrm{cm}^{\wedge} 2$

Fig. 4 Comparison of two-phase flow patterns in vapor-venting and conventional channels for various heat fluxes (perspective view) 


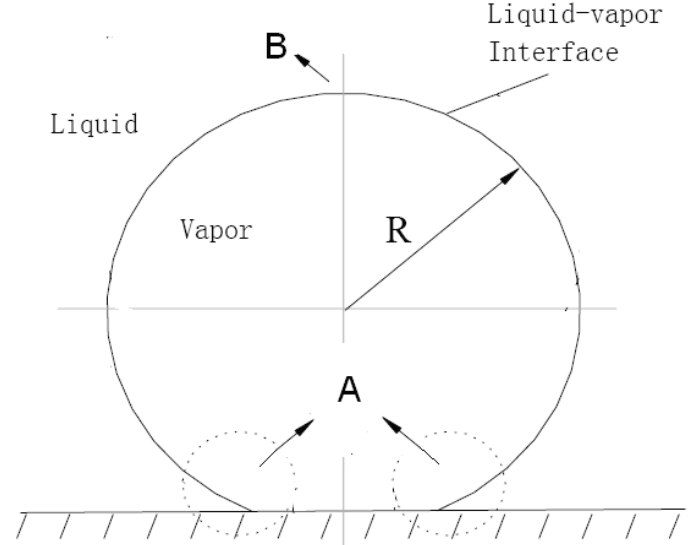

Fig. 5 Equilibrium between condensation and evaporation on a bubble interface during the nucleate boiling

The flow pattern for an intermediate heat flux of $18 \mathrm{~W} / \mathrm{cm}^{2}$ is shown in Fig.4(b). A raised heat flux causes the bulk liquid temperature to grow at a faster rate, yielding a larger equilibrium bubble size as opposed to that in case 1. The simulation clearly shows that the vapor venting process (Fig.4(b1) 4(b3)) can effectively limit the buildup of the two-phase mixture quality in the downstream and hinder the formation of the elongated liquid slug and the annular flow, which appears in the downstream region of the conventional channel (Fig.4 (b4) 4(b6)) leaving only a small fraction of cross section for liquid transfer. A similar flow pattern was also observed in a microchannel condensation (Fang et al., 2010). The long slug in the conventional channel is initially lubricated by thin liquid films separating the vapor core with the channel wall. The excessively long slug eventually leads to the liquid film rupture, yielding dry-out regions (Fig.4(b5) 4(b6)). The dry-out issue was also addressed by Chang and Pan (2007), who stated that the dominant effect of the surface tension force over the buoyancy and lift forces at small scale exacerbates the dry-out condition. In the vapor-venting channel, however, the elongated slug is absent and the dry-out may never occur. The simulated flow pattern here agrees with the experimental observation reported previously (Zhang et al.,2005; Alexander and Wang, 2008).

Figure 4(c) shows the flow pattern for a high heat flux of $23 \mathrm{~W} / \mathrm{cm}^{2}$. Such high heat flux generates the vapor at a rate exceeding the venting capacity of the channel. Consequently, the excessive vapor accumulation and the slug/annular flow pattern appear in both channels. Unlike those in case 1 and case 2, the flow patterns shown in Fig.4c do not indicate a quasi-static state. Instead, the quality of mixture continuously grows with the time and the dryout region expands across the whole channel.

A single bubble venting process is illustrated in Fig.6 for heat flux of $10 \mathrm{~W} / \mathrm{cm}^{2}$. The bubble initially grows to the channel size and comes into contact with the membrane surface (Fig.6(a)). The venting process starts with the rupture of the liquid film separating the vapor and membrane, and the surface-tension-induced high pressure in the bubble drives the vapor into the membrane region through a neck (Fig.6(b)). The vapor flow experiences a high resistance in the membrane, causing the vapor flow to divert to the sidewalls and expanding the venting neck sidewards, thus forming a cylindrical vapor column (Fig.6(c) 6(d)). A pair of vortex was also induced by the expansion process (Fig.6(c)). The sideward expansion eventually transforms the vapor column into a semi-spherical bubble attached to the membrane surface, and the venting area and venting rate reach a peak value, as is evidenced by the velocity field in the membrane region in Fig.6(e). The continuous venting process causes the bubble to shrink (Fig.6(f) $\sim 6(\mathrm{~g})$ ) and finally disappear, leaving the channel with a big circulation of liquid flow, which is eventually dampened by the viscous resistance (Fig.6(h)). The simulated venting process is similar to what was observed by David et al. (2009) in a adiabatic water-air system, as shown in Fig.7. The bubble venting duration is around $0.5 \mathrm{~ms}$ in both experiment and simulation.

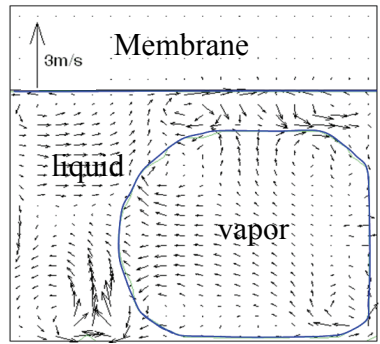

(a) $23.482 \mathrm{~ms}$

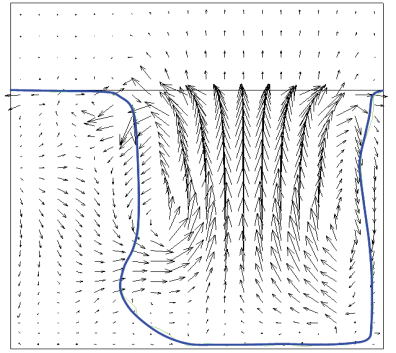

(c) $23.570 \mathrm{~ms}$

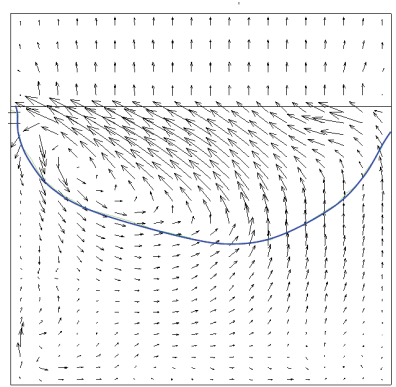

(e) $23.682 \mathrm{~ms}$

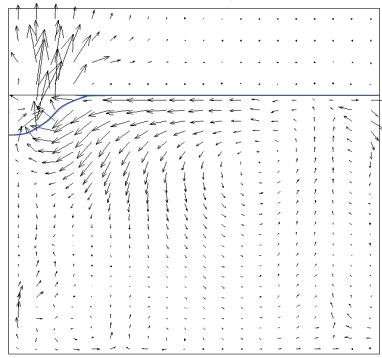

(g) $23.782 \mathrm{~ms}$

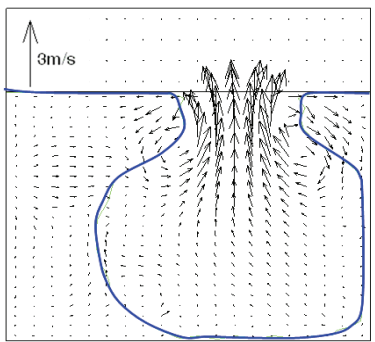

(b) $23.545 \mathrm{~ms}$

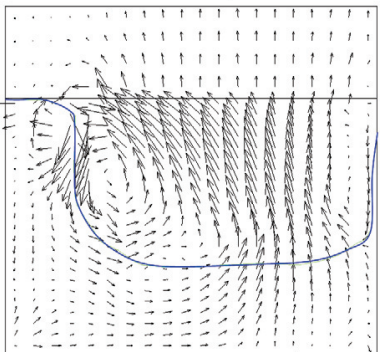

(d) $23.632 \mathrm{~ms}$

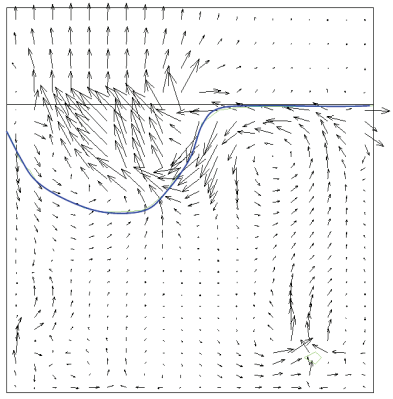

(f) $23.732 \mathrm{~ms}$

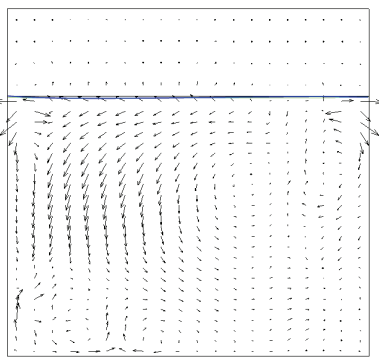

(h) $23.832 \mathrm{~ms}$
Fig. 6 Velocity field of a typical bubble-venting process. The cross section of the membrane-channel assembly is shown and blue curves indicate the vapor-liquid interface.

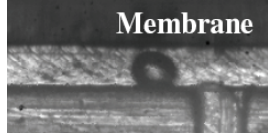

$0 \mathrm{~ms}$

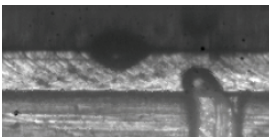

$0.45 \mathrm{~ms}$

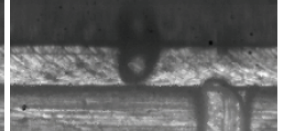

$0.15 \mathrm{~ms}$

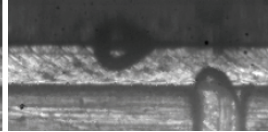

$0.3 \mathrm{~ms}$

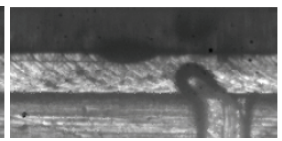

$0.6 \mathrm{~ms}$

Fig.7 Experimental observation of a venting process in a waterair adiabatic system at a liquid flowrate of $0.26 \mathrm{ml} / \mathrm{min}$ and an air flow rate of $0.23 \mathrm{ml} / \mathrm{min}$ (David et al., 2009). 


\subsection{Pressure Distribution}

Figure 8 shows a snapshot of the pressure contour when the channel and the membrane are occupied by the single liquid and the single vapor phase, respectively. A capillary pressure jump across the membrane-channel interface is evident, which prevents the liquid phase from leaking into the membrane. Serving as a no-slip solid wall to the liquid phase, the hydrophobic membrane allows the development of the standard Poiseuille flow in the channel, leading to a linear pressure drop in the longitudinal direction.

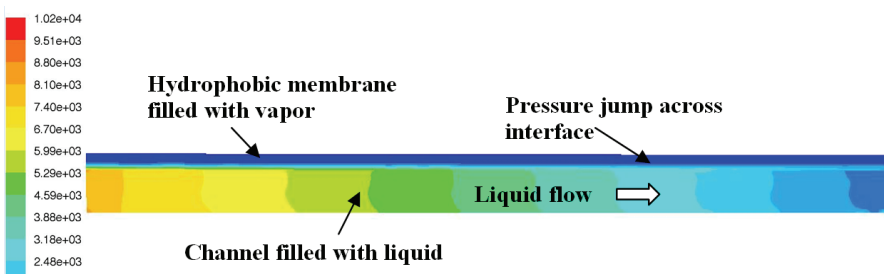

Fig.8 Calculated pressure contour in the membrane and the microchannel prior to onset of boiling

\subsubsection{Reduction of Average Pressure Drop}

Figure 9 shows the comparison of the pressure drop between the conventional channel and the vapor-venting channel for various heat fluxes. For a heat flux lower than $6 \mathrm{~W} / \mathrm{cm}^{2}$, the channel is occupied by the liquid single phase and both types of channel yield identical pressure drops. As the heat flux exceeds $6 \mathrm{~W} / \mathrm{cm}^{2}$, the boiling occurs, starting near the channel outlet and propagating upstream with an increase in heat flux. As discussed by Zhang et al. (2002), after the onset of boiling, the confined geometry of the microchannel without vapor-venting causes the generated vapor phase to rapidly fill and clog the channel, leaving only a small fraction of the cross section available for liquid transfer. This added friction dramatically lifts the pressure drop. Our simulation shows that the pressure drop increases with the heat flux at an approximate rate of $160 \mathrm{~N} / \mathrm{W}$ within the range of $6 \mathrm{~W} / \mathrm{cm}^{2}$ to $25 \mathrm{~W} / \mathrm{cm}^{2}$. The result is consistent with the prediction of the steady-state model proposed by Koo et al. (2001), which was also used by Zhang et al. (2002), Zhang et al. (2005) and David et al. (2007) for the comparison with experimental measurement.

For the vapor-venting microchannel, the pressure drop initially increases with the heat flux after the onset of boiling at a rate similar to that of the conventional channel, as shown in the region A of Fig.9. However, the curve starts to flatten out with a further increase in the heat flux beyond around $10 \mathrm{~W} / \mathrm{cm}^{2}$ (Fig.9 region B). The pressure drop becomes insensitive to the rise of heat flux in the high heat flux regime, which clearly shows the advantage of the vaporventing channel over the conventional channel in terms of the pressure drop reduction. The initial increase of the pressure drop in region $\mathrm{A}$ is attributed to the presence of moving small bubbles which are not big enough to contact the membrane surface and escape. Those bubbles are convected downstream, partially blocking the channel and increasing the overall pressure drop. An optimization of the channel structure would facilitate the escape of those small bubbles and may relieve the pressure buildup in region A. Instead of the slug and annular flow in the conventional channel, the high heat flux in the vapor-venting channel only induces a massive vaporescaping process and the pressure stops further increasing due to the limited mixture quality buildup.

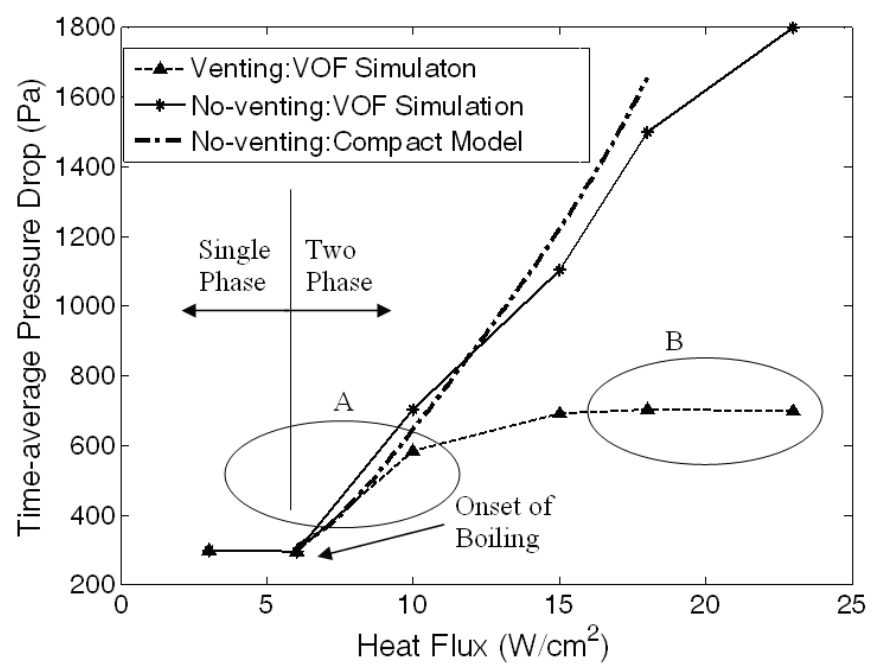

Fig.9 Comparison of relationship between the mean pressure drop and the heat flux for two types of microchannels

\subsubsection{Pressure Fluctuation}

Figure 10 illustrates the temporal pressure evolution for both types of channels at a heat flux of $11 \mathrm{~W} / \mathrm{cm}^{2}$. The pressure fluctuation appears in both conventional and vapor-venting channels. These pressure fluctuations, which are observed mainly in microchannels, are primarily due to bubble formation, expansion and departure (Zhang et al., 2002; Zhang et al., 2005) The present simulation also predicts pressure fluctuation on the order of a few hundred $\mathrm{Pa}$ in a 5 $\mathrm{mm}$ long channel, though their reported frequency is limited by the computational time step size and thus lacks physical meaning.

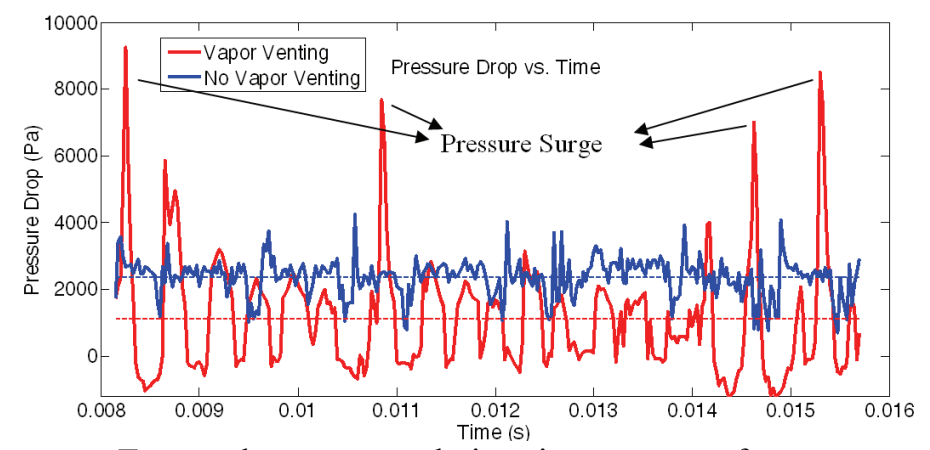

Fig.10 Temporal pressure evolutions in two types of microchannels (heat flux: $11 \mathrm{~W} / \mathrm{cm}^{\wedge} 2$ ). Significant pressure surges are present in the vapor-venting channel.

In the vapor-venting channel, bubbles still experience an initial expansion. However, the expansion process is halted when the bubble reaches the channel size. Hence, the high frequency component of the pressure fluctuation also exists in the vapor-venting channel, although the average amplitude is lower than that in the conventional channel. In addition to the high frequency component, the simulation also reveals a new pressure fluctuation mode with a low frequency of around $1 \mathrm{kHz}$ and a high amplitude. The peak amplitude of this low frequency fluctuation can be up to several thousand $\mathrm{Pa}$, as shown by spikes on the red curve in Fig.10. A synchronized examination of the flow pattern and the pressure time series implies that the pressure surge is induced by the final stage of the bubble venting, as shown in Fig.11. During the venting process, the liquid cavity caused by the bubble shrinkage is filled by upstream liquid, which leads to a discontinuity in the liquid flowrate at the venting site (Fig.11 (A) 11(B)). The velocity field shown in Fig.12 
clearly shows the abrupt flowrate drop at the venting site. The flowrate immediately downstream of the venting site gets vanishingly small and in some extreme cases may even result in reverse flow. Due to the incompressibility of the liquid however, the completion of the venting process dictates an immediate removal of such flowrate discontinuity, leading to an abrupt deceleration of the upstream liquid and an acceleration of the downstream liquid. Consequently, the induced inertial force causes a pressure surge in the channel (Fig.11(C)). The amplitude of the pressure surge is negatively correlated with the distance between the channel inlet and the venting site, and the fluctuation frequency is dictated by the number of bubbles undergoing the venting process per unit time. The overall pressure fluctuation in the vapor-venting channel is the superposition of the bubble-expansion-induced high frequency component and the flowrate-discontinuity-induced low frequency component.

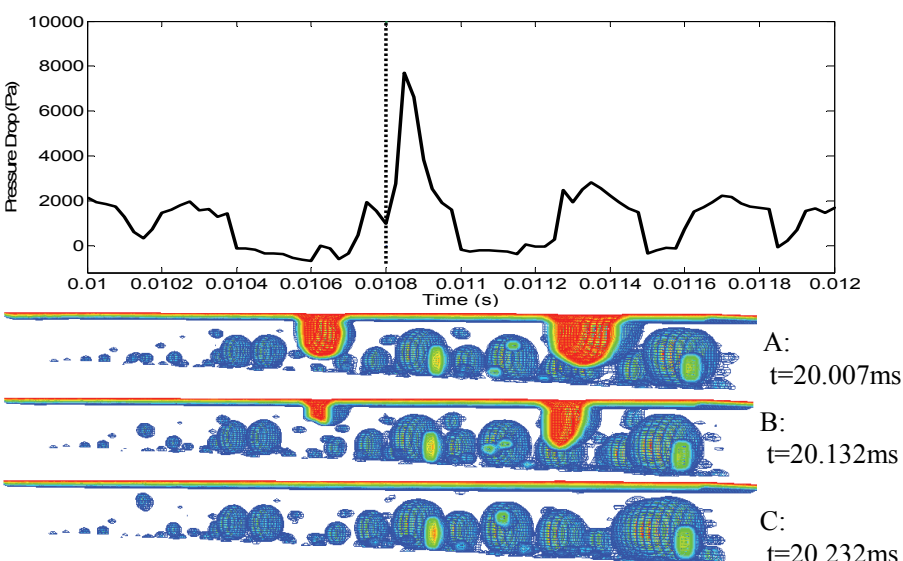

Fig.11 Pressure surge process versus vapor-venting flow patterns (heat flux: $10 \mathrm{~W} / \mathrm{cm}^{\wedge} 2$ )

Although the simulation shows that the vapor-venting mechanism may exacerbate rather than mitigate the pressure fluctuation issue in a single channel, it may still suppress the pressure fluctuation in a multi-channel system, considering the vapor-venting mechanism can effectively alter the demand curve which is responsible for the pressure fluctuation caused by Ledinegg instability in an ordinary multi-channel system without vapor-venting.

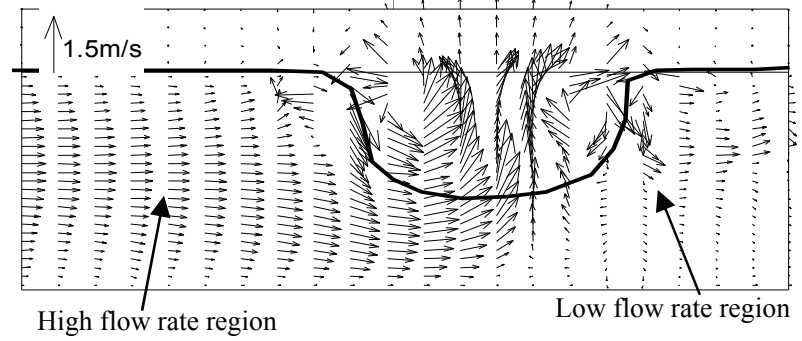

Fig.12 Velocity discontinuity during venting. A transition from the high flow rate region to the low flow rate region occurs during the bubble venting process.

\subsection{Temperature Distribution}

In addition to the pressure drop reduction, another major advantage of the vapor-venting microchannel, as suggested by Zhou et al. (2006), lies in its mitigation of the local dry-out which leads to hotspots on the chip. Our simulation firmly supports this argument. Figure 13 shows the temperature distribution along the channel bottom subject to a variety of heat fluxes, with the corresponding flow patterns illustrated. In all cases, the temperature quickly rises from the inlet liquid temperature $370 \mathrm{~K}$ to the saturation point where boiling occurs.

For a low heat flux of $10 \mathrm{~W} / \mathrm{cm}^{2}$, the temperature profile in both channels remains flat over the whole boiling region, and the supplied heat is purely absorbed by the phase change process. Similar temperature profile was also observed by Zhang et al. (2002) in the silicon microchannel heat sink. Although the conventional channel and the vapor-venting channel are dominated by the bubbly-slug flow (Fig.13(A)) and pure bubbly flow (Fig.13(B)), respectively, the existence of the liquid film on the channel wall in both channels sustains a continuous evaporation and a stable wall temperature is maintained for both flow patterns.

As the heat flux increases to $18 \mathrm{~W} / \mathrm{cm}^{2}$, the vapor venting process starts to play a critical role in preventing the formation of the elongated liquid slug and maintaining the liquid film evaporation (Fig.13(C)). In contrast, the excessive vapor accumulation in the conventional channel rapidly creates an elongated vapor slug in the upstream and an annular flow in the downstream (Fig.13(D)), where the liquid film rupture and the local dry-out appears on the channel bottom. When dry-out occurs, the thermal resistance between the channel wall and the liquid absorbing the heat surges drastically, and the heat removal capacity in those corresponding areas is reduced by several orders of magnitude (Chang and Pan 2007). Consequently, the bottom temperature in the conventional channel surges to around $420 \mathrm{~K}$, which is in sharp contrast to the almost unchanged temperature in the vapor-venting channel.

By further ramping the heat flux up to $23 \mathrm{~W} / \mathrm{cm}^{2}$, we may make the vapor generation rate significantly exceed the vapor removal capacity of the membrane. Consequently, an annular/stratified flow accompanied by the dry-out occurs in both channels, leading to a drastic temperature surge (Fig.13(E) 13(F)).

The simulation shows that the vapor-venting mechanism can dramatically suppress the local dry-out, and hence substantially enhance the critical heat flux that a microchannel heat sink can operate at.

\subsection{Impact of Membrane Surface Hydrophobicity}

Recently, a number of experimental studies were conducted to study the air-venting process in the microchannel, which bears some resemblance with the present vapor-venting microchannel. However, different flow patterns were reported by those studies. David et al. (2009) reported the stratified flow at a moderate to high air flow rate in the copper microchannel with a porous hydrophobic Teflon membrane wall. The contact angle at the membrane wall is $140^{\circ}$. In contrast, Alexander et al. (2008) tested the air-venting process in the microchannel with parallel breather ports located at the sidewall. The breather ports are coated with a Silane SAM rendering a contact angle of $116.3^{\circ}$, while the channel wall is kept moderately hydrophilic with a contact angle around $90^{\circ}$. Instead of the stratified flow, the study reported the slug flow pattern during the venting process. Similar flow pattern was also reported by the Meng et al. (2004) using a Teflon membrane breather with the surface contact angle of $60^{\circ}$ to $67^{\circ}$ for the methanol. The discrepancy is possibly attributed to the variance in the wettability of the breather's surface, which is hydrophobic (David et al., 2009) and semi-hydrophilic (Meng et al., 2004; Alexander et al., 2008).

To verify this argument, the vapor-venting process in the channel with various membrane surface contact angles is simulated. The resulting flow patterns are shown in Fig.14. The bubbly flow (Fig.14(a)) and elongated slug flow (Fig.14(c)) are observed in channels with moderately hydrophilic membrane and hydrophilic membrane, respectively, which is similar to those reported previously (Alexander et al., 2008). In contrast, the bubble in the superhydrophobic case (Fig.14(b)) tends to spread on the membrane surface and form a vapor buffer layer. 


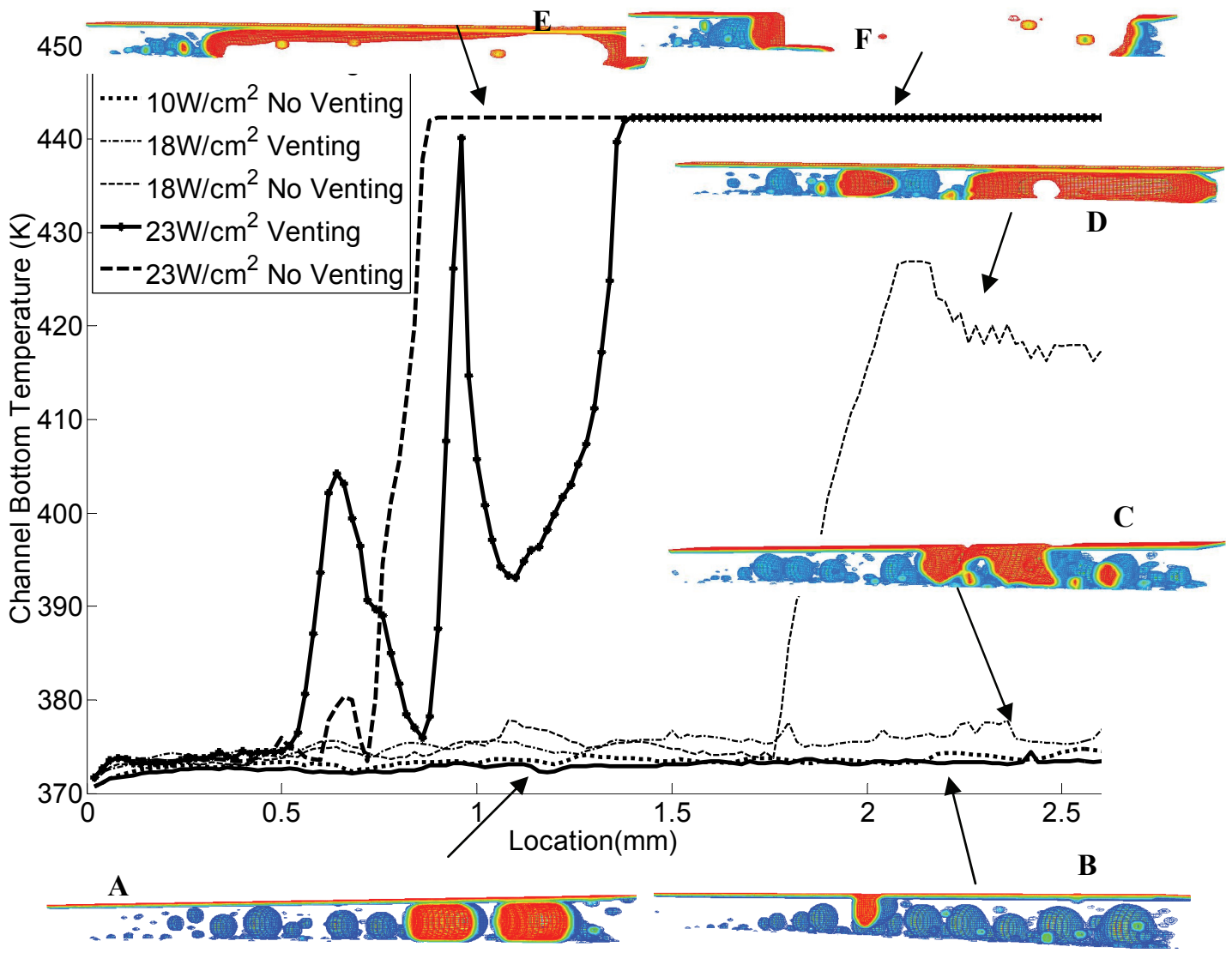

Fig.13 Temperature distribution along the bottom of the channel for two types of microchannels and various heat fluxes.

The simulation shows that the formation of the vapor buffer layer significantly increases the contact area between the vapor and membrane, therefore enhancing the vapor-venting rate, whereas the hydrophilic membrane surface in Fig.14(c) minimizes the vapormembrane contact area and almost completely inhibits vapor venting.

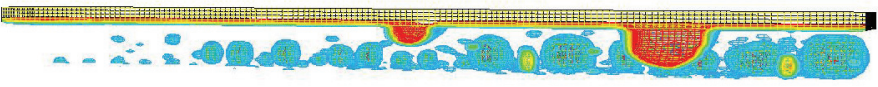

(a) Moderately hydrophobic membrane surface (114.5 deg)

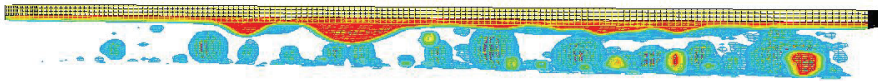

(b) Hydrophobic membrane surface (contact angle: 179 deg)

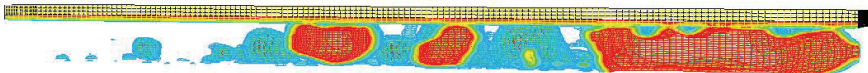

(c) Hydrophilic membrane surface (contact angle: $28.3 \mathrm{deg}$ )

Fig.14 Impact of membrane surface hydrophobicity on the vaporventing flow pattern $\left(10 \mathrm{~W} / \mathrm{m}^{2}\right)$

\subsection{Impact of Membrane Condensation}

David et al. (2008) tested the operation of the vapor-venting copper heat exchangers with built-in PTFE membranes and found that in certain conditions the device fails to show the improvement in the pressure drop, implying that "the membrane acts like a solid wall". Due to the lack of the visualization capability, such hypothesis has yet to be confirmed.

To verify this argument, we artificially induce the condensation in the membrane by applying a heat sink of $0.5 \mathrm{~W} / \mathrm{cm}^{2}$ to the top surface of the membrane. The impact of the membrane condensation on the flow pattern is illustrated in Fig.15, which clearly shows that the condensed liquid in the membrane can degenerate the vaporventing channel into a conventional channel with a hydrophobic top surface (Fig.15(a)). Due to the low permeability of the membrane to the liquid water ( $4 \mathrm{e}-22 \mathrm{~m}^{2}$ in the present study) the condensed water is retained in the membrane and blocks the passage in the porous structure available for vapor venting. The membrane condensation may be mitigated by vaporizing and removing the water from the membrane.

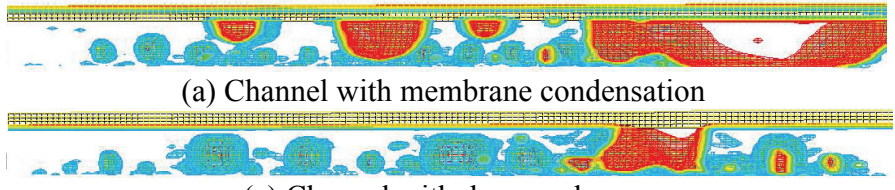

(a) Channel with dry membrane

Fig.15 Impact of membrane condensation on the vapor-venting process. Note how membrane condensation prevents vapor from escaping

\section{CONCLUSIONS}

The present study numerically simulates the $3 \mathrm{D}$ transient vaporventing process in a microchannel with a hydrophobic porous membrane using the VOF method along with a capillary force model. A comparison is provided between the vapor-venting channel and the conventional channel for the two-phase flow patterns, the pressure drop, and the temperature profile. The effects of the membrane surface wettability and the membrane condensation on the heat exchanger performance are also discussed. We conclude: 
1. Over a full range of the heat flux below the critical value, the vapor-venting channel yields a significantly smaller pressure drop as opposed to the conventional microchannel. As the heat flux exceeds the critical value, both channels are occupied by the annular flow and yield similar pressure drop.

2. The temporal pressure fluctuation in the vapor-venting channel is the superposition of a high-frequency-low-amplitude component induced by the bubble expansion and a low-frequency-highamplitude component caused by the bubble venting, the latter being absent in the conventional channel.

3. Vapor-venting mechanism can significantly inhibit the liquid film rupture and local dry-out, a phenomenon commonly appearing in the conventional microchannel when heat flux is high. The temperature profile remains stable in the vapor-venting channel for a full range of heat fluxes below the critical value.

4. Superhydrophobic membrane surface induces the formation of a buffer vapor layer, which enhances the vapor-venting efficiency due to the increased vapor-venting area. The hydrophilic membrane surface significantlly compromises the device performance due to the diminished contact area between vapor and membrane.

5. The vapor-venting process suffers from the condensation in the membrane. Possible solutions include maintaining the temperature of the membrane and finding a highly permeable membrane with a finepore, superhydrophobic surface to create a high capillary force.

The present numerical model may assist in the design and optimization of the next generation vapor-venting heat exchanger with an enhanced heat removal capability.

\section{REFERENCES}

Alexander, B.R., and Wang, E.N., 2008. "Design of a Microscale Breather for Two-phase Thermal Management Devices", IMECE 2008-68898, Proceedings of 2008 ASME International Mechanical Engineering Congress and Exposition, Boston MA.

Brackbill, J.U., Kothe, D.B., and Zemach, C., 1992, “A Continuum Method for Modeling Surface Tension," J. Comput. Phys., 100, 335354.

\section{doi:10.1016/0021-9991(92)90240-Y}

Chang, K., and Pan, C., 2007, "Two-phase Flow Instability for Boiling in a Microchannel Heat Sink," International Journal of Heat and Mass Transfer, 50, 2078-2088

doi:10.1016/j.ijheatmasstransfer.2006.11.014

David, M.P., Khurana, T., Hidrovo, C., Pruitt, B.L., and Goodson, K.E., 2007, "Vapor-venting, Micromachined Heat Exchanger for Electronics Cooling," Proceedings of IMECE, Seattle, WA.

David, M.P., Marconnet, A., and Goodson, K.E., 2008, "Hydrodynamic and Thermal Performance of a Vapor-venting, Micromachined Copper Heat Exhcnager," Proceedings of ICNMM, Darmstadt, Germany.

David, M.P., Steinbrenner, J., Miler, J., and Goodson, K.E., 2009 , "Visualization and Analysis of Venting from a Single Microchannel Two-phase Copper Heat Exchanger," Proceedings of InterPack, San Francisco, California.

Figus, C., Bray, Y., Bories, S., and Prat, M., 1999, "Heat and Mass Transfer with Phase Change in a Porous Structure Partially Heated: Continuum Model and Pore Network Simulations," Int. J. Heat and Mass Transfer, 42, 2557-2569

doi: 10.1016/S0017-9310(98)00342-1

Fang, C., Carlos, C., Wang, F., Eaton, J., and Goodson, K., 2008, "3D Numerical Simulation of Contact Angle Hysteresis for Microscale Two-phase Flow," Int. J. Multiphase Flow, 34, 690-705

doi:10.1016/i.ijmultiphaseflow.2007.08.008
Fang, C., Steinbrenner, J., Wang, F., and Goodson, K., 2010, "Impact of Wall Hydrophobicity on Condensation Flow and Heat Transfer in Silicon Microchannels," J. Micromech. Microeng., 20, 045018 doi: 10.1088/0960-1317/20/4/045018

Fang, C., David, M., Wang, F., and Goodson, K., 2010, "Influence of Film Thickness and Cross-sectional Geometry on Hydrophilic Microchannel Condensation," Int. J. Multiphase Flow, in press doi:10.1016/ j.ijmultiphaseflow.2010.04.005

Ganesh, R.K., Faghri, A., and Hahn, Y., 1997, “A Generalized Thermal Modeling for Laser Drilling Process: Part I- Mathematical Modeling and Numerical Methodology," Int. J. Heat Mass Transfer, 40, 3351-3360 doi:10.1016/S0017-9310(96)00368-7)00368-7

Garimella, S., 2002, “An Experimentally Validated Model for Twophase Pressure Drop in the Intermittent Flow Regime for Circular Microchannels," J. Fluids Eng., 124, 205-214

doi:10.1115/1.1601258

Garimella, S., and Singhal, V., 2004, "Single Phase Flow and Heat Transport and Pumping Considerations in Microchannel Heat Sinks," Heat Transfer Engineering, 25, 15-25

doi:10.1080/01457630490248241

Issa, R.I., Gosman, A.D., and Watkins, A.P., 1991, "The Computation of Compressible and Incompressible Recirculating Flows by a Noniterative Implicit Scheme," J. Comput. Phys., 93, 388-410. doi:10.1016/0021-9991(86)90100-2

Kennedy, J.E., Roach, G.M., Dowling, M.F., Abdel-Khalik, S.I., Ghjaasiaan, S.M., and Jeter, S.M., 2000, "The Onset of Flow Instability in Uniformly Heated Horizontal Microchannels," J. Heat Transfer, 122, 118-125

doi: $10.1115 / 1.521442521442$

Koo, J., Jiang, L., Zhang, L., Zhou, P., Banerjee, S.S., Kenny, T.W., Santiago, J.G., and Goodson, K.E., 2001, "Modeling of Two-phase Microchannel Heat Sinks for VLSI Chips," Proceedings of Int. MEMS Workshop, 422-426

Kandlikar, S., and Grande, W., 2004, "Evaluation of Single Phase Flow in Microchannels for High Heat Flux Chip CoolingThermohydraulic Performance Enhancement and Fabrication Technology," Heat Transfer Engineering, 25, 5-16 doi:10.1080/0145763049051977201457630490519772

Kaya, T., and Goldak, J., 2006, "Numerical Analysis of Heat and Mass Transfer in the Capillary Structure of a Loop Heat Pipe," Int. J. Heat and Mass Transfer, 49, 3211-3220

doi:10.1016/j.ijheatmasstransfer.2006.01.028

Lee, W.H., 1980, "A Pressure Iteration Scheme for Two-phase Flow Modeling," Multiphase Transport Fundamentals, Reactor Safety, Applications, Hemisphere Publishing, Washington, DC

Li, Z.M., 2002, "Mass Transfer Behavior at Bubble Surface During Nucleate Boiling," Heat and Mass Transfer, 38, 433-439

doi:10.1007/s002310100253

Lee, P., Tseng, and F., Pan, C., 2004, "Bubble Dynamics in Microchannels. Part I: Single Microchanel," Int. J. Heat and Mass Transfer, 47, 5575-5589

doi:10.1016/i.ijheatmasstransfer.2004.02.031

Meng, D.S., Cubaud, T., Ho, C., Kim, C., 2004, "A Membrane Breather for Micro Fuel Cells with High Concentration Methanol," Proc. Tech. Dig. Solid-state Sens., Actuator, Microsyst. Workshop, Hilton Heat Island, SC, 141-144 
Meng, D.S., Cubaud, T., Ho, C., and Kim, C., 2007, "A Methanoltolerant Gas-venting Microchannel for a Microdirect Methanol Fuel Cell," Journal of Microelectromechanical Systems, 16, 1403-1410

Qu, W., and Mudawar, I., 2002, "Experimental and Numerical Study of Pressure Drop and Heat Transfer in a Single-phase Microchannel Heat Sink," Int. J. Heat and Mass Transfer, 45, 2549-2565 doi:10.1016/S0017-9310(01)00337-4

Ren, C., Wu, Q., and Hu, M., 2007, "Heat Transfer with Flow and Evaporation in Loop Heat Pipe's Wick at Low or Moderate Heat Fluxes," Int. J. Heat and Mass Transfer, 50, 2296-2308

doi:10.1016/j.ijheatmasstransfer.2006.10.029

Tuckerman, D.B., and Pease, R.F.W., 1981, "High-performance Heat Sinking for VLSI," IEEE Electron Device Letters, Vol.EDL2, No.5, 126

Takata, Y., Shirakawa, H., Sasaki, H., Kuroki, T., and Ito, T., 1999, "Numerical Analysis of Rapid Solidification in a Single Roller Process," Heat Transfer - Asian Research, 28, 34-49 doi:10.1002/(SICI)1523-1496(1999)28:1<34::AID-

HTJ4>3.0.CO;2-0

Welch, S.W.J., and Wilson, J., 2000, "A Volume of Fluid Based Method for Fluid Flows with Phase Change," Journal of Computational Physics, 160, 662-682 doi:10.1006/jcph.2000.6481
Youngs, D.L., 1982, “Time-dependent Multi-material Flow with Large Fluid Distortion," in: Numerical Methods for Fluid Dynamics, K.W. Morton and M.J. Baines (eds.), New York Academic Press, 273-285.

Yang, Z., Peng, X.F., and Ye, P., 2008, "Numerical and Experimental Investigation of Two Phase Flow During Boiling in a Coiled Tube," International Journal of Heat and Mass Transfer, 51, 1003-1016 doi:10.1016/j.ijheatmasstransfer.2007.05.025

Zhang, Y., Faghri, A., and Shafii, M.B., 2001, "Capillary Blocking in Forced Convective Condensation in Horizontal Miniature Channel," Journal of Heat Transfer, 123, 501-511 doi:10.1115/1.13518081.1351808

Zhang, L., Koo, J., Jiang, L., Asheghi, M., Goodson, K.E., Santiago, and J.G., Kenny, T.W., 2002, "Measurements and Modeling of Twophase Flow in Microchannels with Nearly Constant Heat Flux Boundary Conditions," Journal of Microelectromechanical Systems, 11, $12-19$ doi:10.1109/84.982858

Zhang, L., Wang, E.N., Goodson, K., and Kenney, T., 2005, "Phase Change Phenomena in Silicon Microchannels," International Journal of Heat Mass Transfer, 48, 1572-1582

doi:10.1016/j.ijheatmasstransfer.2004.09.048

Zhou, P., Goodson, K.E., and Santiago, J., 2006, U.S. Patent 6, 994, $151 \mathrm{~B} 2$ 